Mirai. Estudios Japoneses

ISSN-e: 2531-145X

http://dx.doi.org/10.5209/MIRA.60496

\title{
El viaje en busca de Ono no Komachi
}

\author{
Kayoko Takagi Takanashi ${ }^{1}$
}

Fechas

Resumen. Ono no Komachi es una escritora cuyas obras e imagen han ofrecido a lo largo de la historia múltiples manifestaciones, tanto para la cultura en general como para los asuntos más cotidianos. A partir de una búsqueda de lugares que reclaman su autenticidad como escenario de su vida y leyendas quedaría patente una asimilación de la cultura y de las tradiciones antiguas en tales lugares. Esta asimilación se debe a una apreciación profunda del valor artístico y estético de la figura de Komachi. Como consecuencia, se origina un movimiento de gente para visitar esos lugares. El nombre de Komachi está siendo utilizado como denominación comercial de un nuevo shinkansen a Akita, región que reclama ser su lugar de origen. A través de la labor documental y de un trabajo de campo, este estudio intentará demostrar la importancia de la figura de Komachi en la historia cultural de Japón y su efecto socio-cultural en el Japón actual, particularmente, en la región de Akita.

Palabras claves: Ono no Komachi; imagen del lugar; leyendas; historia cultural.

\section{[en] The journey in search of Ono no Komachi}

Abstract. Ono no Komachi is a literary figure whose works and image have been present throughout time in multiple manifestations, linked not only with general culture but also with more quotidian affairs. The search for places that claim their authenticity as locations related to her life and legends reveals how culture and old traditions have become assimilated to those places. This assimilation is due to a profound appreciation of the artistic and esthetic values of the figure of Komachi. This has originated a movement of people that goes to visit these places. The name Komachi is being used as a commercial brand name for a new shinkansen to Akita, region that claims to be her birthplace. Through documentary research and field work, this study aims to prove the importance of the name Komachi in Japanese cultural history and its socio-cultural results in contemporary Japan, particularly in the region of Akita.

Keywords: Ono no Komachi; Image of the place; legends; cultural history.

Sumario: 1. La lectura y el viaje como método de trabajo. 2. Obras literarias sobre Ono no Komachi. 3. Dispersión geográfica del nombre del clan Ono y de Komachi. 4. Ogachi hoy. 5. El desarrollo de leyendas y sus memorias. 6 . Conclusion.

Cómo citar: Takagi Takanashi, K. (2018). El viaje en busca de Ono no Komachi, en Mirai. Estudios Japoneses 2(2018), 67-79.

1 Centro de Estudios de Asia Oriental. Universidad Autónoma de Madrid.

kayoko.takagi@uam.es 


\section{La lectura y el viaje como método de trabajo}

El propósito de este artículo es demostrar, por un lado, la importancia de la figura de la poetisa Ono no Komachi en la historia cultural de Japón y, por otra, su efecto socio-cultural que deriva de diferentes obras literarias relacionadas con ella. Hoy la imagen legendaria que se ha creado sobre su persona se focaliza con más insistencia en dos lugares del país: Kioto, la corte imperial donde desarrolló su actividad artística, y la región norteña de Akita, parte de la zona antiguamente llamada Mutsu, que reclama ser lugar de su origen. Entre estos dos lugares, a fin de cumplir con el objetivo del trabajo, he escogido el último como enfoque geográfico por considerar que Akita representa un lugar de la periferia que puede arrojar otro tipo de novedosa luz al estudio.

La metodología del trabajo consiste, por tanto, en la lectura crítica de las poesías de Komachi como en el estudio de otras obras literarias relacionadas con su figura $\mathrm{y}$, después, en proceder a la búsqueda de los fenómenos socio-culturales que lleven el nombre de Komachi en la actualidad. Se han tomado en cuenta, en primer lugar, obras de investigación como fuentes primarias que se mencionarán más adelante y, en segundo lugar, diversos fenómenos turísticos, culturales, así como también promociones comerciales populares en el Japón de hoy.

En cuanto a la búsqueda de elementos que indican la influencia de la imagen histórica de Komachi, he realizado un desplazamiento como trabajo de campo con el propósito de identificar los lugares y ruinas in situ que anuncian hoy la relación directa con la vida de la famosa poetisa de Heian. Concretamente, las visitas se centraron alrededor de la villa de Ogachi, cerca de la ciudad de Yuzawa, en la prefectura de Akita. [fig. 1]

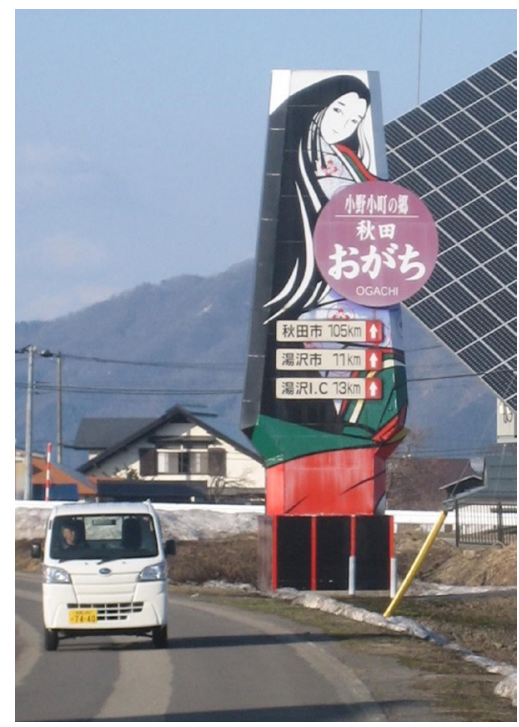

Fig. 1: Komachi en Ogachi, Señal indicadora de la villa. Ayuntamiento de Ogachi. El indicador representa la imagen de una Komachi imaginaria con la inscripción traducida: "Pueblo de Ono no Komachi, Akita, Ogachi", escrito en hiragana y en romaji. Se leen las distancias desde el lugar: "A la ciudad de Akita, $105 \mathrm{Km}$, a la Ciudad de Yuzawa, $11 \mathrm{Km}$ y a la autovía de Yuzawa, 13 Km.” (C) 2016, Kayoko Takagi. 
El tema de este artículo, "el viaje en busca de Ono no Komachi”, comenzó por la lectura del libro Ono no Komachi densetsu no tanjō (小野小町伝説の誕生), de Nishiki Hitoshi. ${ }^{2}$ El autor es natural de Ogachi, una comarca cerca de Yuzawa, en la prefectura de Akita, donde se guardan monumentos y ruinas relacionadas con la vida de la poetisa. Dicho autor realizó una investigación exhaustiva durante largos años sobre el tema y tuvo acceso a los libros de guerra del género gunki monogatari de la zona de los siglos XVI y XVII ${ }^{3}$ y también a las primeras crónicas de viaje que se refieren a esa legendaria relación, redactadas por Sugae Masumi (1754-1829), ${ }^{4}$ funcionario inspector de la zona, destinado a la región a principios del siglo XIX.

Además, el libro de Nishiki narra con gran minuciosidad las huellas folklóricas, los lugares, los objetos y las representaciones atribuidas a la vida de Komachi. Como conclusión, esta investigación nos demuestra las posibles causas y razones por las que las leyendas de Komachi se han conformado en la memoria colectiva de la zona en la forma que hoy conocemos.

En cuanto a las obras literarias inspiradas en la poetisa, anteriormente había realizado estudios sobre su figura literaria con la traducción al español de una obra representativa del teatro nō, Kayoi Komachi (通い小町), ${ }^{5}$ una de las siete piezas denominadas Nana komachi (七小町, Siete piezas de Komachi), escrita por Zeami. ${ }^{6}$ Se ha realizado asimismo la lectura crítica de sus obras de waka que aparecen en Kokinwakashū (古今和歌集), como obras que ofrecen mayor credibilidad sobre su autoría. ${ }^{7}$ Sin embargo, hay que señalar que el inicial estudio de dichas obras no quedó solo en el terreno literario. Fue abriendo un abanico de elementos muy dispares sobre su vida e imagen histórica en las que no faltan enigmas e imprecisiones. Se puede considerar que este hecho se debe al proceso de legendarización de la poetisa, que parece comenzar prácticamente nada más conocerse su muerte a finales del siglo IX o a principios del X. ${ }^{8}$ La propagación y la evolución posterior de tales relatos a lo largo de la historia arrojan muchos temas interesantes para la investigación. Las obras literarias que hacen referencia a Komachi, o a lo que representa la bella dama de Heian, no han cesado de aparecer hasta nuestros días. Aunque escasean documentos fiables en cuanto a sus datos biográficos, abundan menciones a Komachi en todo tipo de obras tanto clásicas como modernas. En cada etapa de la historia de Japón, su nombre no dejó de sonar, pero como temas de carácter muy diverso.

2 Nishiki, Hitoshi (2004): Ono no Komachi densetsu no tanjō (小野小町伝説の誕誕生). Tokio: Kadokawa sensho n. 364, Kadokawa shoten.

3 Tobe, Ikkansai (2004): Ōu eikei gunki (奥羽永慶軍記), tomo 36. En Nishiki, Hitoshi (2004): Ono no Komachi..., op. cit. p. 16.

$4 \quad$ Sugae Masumi (菅江真澄). Escritor de viajes y estudioso de historia natural dejó unas crónicas sobre la zona en dos escritos: Komahi no furusato (小町のふるさと) y Yuki no dewaji・Ogachigun (雪の出羽路・雄勝郡) de 1822. En Nishiki, Hitoshi. (2004): Ono no Komachi..., op. cit.

5 Takagi, Kayoko y Janés, Clara (traductores) (2008): 9 piezas del teatro NÔ. Madrid: Ediciones del Oriente y del Mediterráneo, pp. 175-193.

6 Ibidem.

7 Se considera que las obras de waka compiladas en Kokinwakashū son las únicas de autoría fidedigna de Komachi que aprueban los filólogos japoneses. Kasahara Hisako (2001): Onoke no on'natachi, Komachi to O-tsū (小 野家の女たち、小町とお通). Tokio: Kanrin shobo, p. 42. Por ello mismo, es importante investigar en profundidad dichos poemas.

8 No podemos determinar las fechas del nacimiento ni de la muerte de la poetisa. Sin embargo, si consideramos la compilación de Kokinwakash $\bar{u}$ en 905 y otros testimonios documentales de la época, es posible trazar su vida vagamente desde mediados del siglo IX a principios del siglo X. 
Señalado cuanto antecede, este artículo no pretenderá abarcar todo lo que sugiere esta diversidad de obras y temas, sino más bien, centrará su atención en el peso de herencia cultural de una poetisa de la era Heian, que da motivos para la promoción de una determinada imagen turística para una región concreta. Como hemos mencionado anteriormente, a fin de comprobar este fenómeno actual de manera crítica, he realizado el viaje a Akita para comprobar in situ en qué términos están materializadas las influencias del valor cultural tradicional en la vida cotidiana actual.

\section{Obras literarias sobre Ono no Komachi}

Existe el término Komachi-mono recogiendo las obras producidas en diferentes épocas inspiradas en Komachi: las ya mencionadas de teatro nō, las de jōruri y kabuki, seguidas de las colecciones de otogizōshi. ${ }^{9}$ En ellas Komachi aparece con múltiples caras. El punto de arranque naturalmente es la imagen de una poetisa de Heian del siglo IX que reúne talento y belleza en la misma persona dentro del seno de la Corte Imperial. Sin embargo, esa fama y popularidad pronto derivan a otra de una "mujer fatal" que causa sufrimiento a los hombres y aparece la imagen de una vieja empobrecida y pordiosera como consecuencia de aquella vida insidiosa de su juventud.

Al respecto, existe una relación interesante con la enseñanza del budismo esotérico, especialmente en la escuela Shingon que fundó Kūkai (774-835). La obra en cuestión, cuya autoría se atribuye a Kūkai, está escrita en forma de poemas en chino y sirvió para propagar la historia de la decadencia de una mujer desde el siglo X en adelante. ${ }^{10}$ Además, encontramos otra obra lírica que se le atribuye con más verosimilitud a Kūkai divulgada como Kusōshi (九想詩, Poemas de nueve aspectos) ${ }^{11}$ que inspiró la producción de Kusōzu (九想図, Dibujos de nueve aspectos). ${ }^{12}$ Se trata de describir el proceso de la corrupción del cadáver en nueve estados hasta convertirse completamente en un esqueleto y, por último, desaparecer de la tierra, con el fin de enseñar la fugacidad de lo físico y el sinsentido del apego a la vida material. Sobre el porqué de ver el nombre de Komachi asociado a este tipo de enseñanza budista, hay varios estudios, pero como muestra el artículo citado de Chin, se ha aprovechado la figura emblemática de género femenino en la historia, que es Komachi, hasta la saciedad para un ejercicio a modo de contemplatio en la religión budista. Lo cierto

9 OtogizŌshi御伽草子. Se trata de colecciones de cuentos populares que empezaron a propagarse desde el período Muromachi.

10 Tochio, Takeshi (ed.) (2009): Tamatsukuri Komachishi Sôsuisho, (玉造小町子壮衰書). Tokio: Iwanami shoten. Está escrito en chino arcaico utilizando una métrica antigua. Históricamente, se creía que este libro fue escrito por el mismo Kūkai (774-835). Sin embargo, se puede refutar este rumor ancestral porque Komachi estuvo activa durante el reinado de Jinmyō (833-850), es decir, más tarde que Kūkai y cronológicamente no es posible atribuirle el título o la autoría de esta obra. Sin embargo, la referencia al nombre de Komachi como mujer decaída hasta extremos se ha utilizado con preferencia en las enseñanzas de la escuela Shingon. Como opina Kasahara, es posible que existiera una versión más antigua y que, posteriormente, por la estrategia de la divulgación, se haya aprovechado el nombre de Komachi.

11 Kusōshi (九想詩). La reproducción de Shusho Kusōshi (1678) se encuentra en Tochio, T. (ed.) (2009): Tamatsukuri Komachishi..., op. cit.

12 Chin, Gail (1998): "The Gender of Buddhist Truth: The Female Corpse in a Group of Japanese Paintings". En Japanese Journal of Religious Studies, Vol. 25, No. 3/4, Nagoya, Nanzan Institute for Religion \& Culture, Fall, pp. 277-317. Entre diferentes producciones bajo el mismo título Kusōzu, el templo Anrakuji de Kioto conserva la obra con el nombre concreto de Kusōzu de Ono no Komachi (小野小町九想図). 
es que en algunas obras de teatro nō, la figura de Komachi se sitúa en el centro para predicar el mensaje religioso de la doctrina mahāyāna. ${ }^{13}$

Así, de la variada imagen histórica de Komachi se pueden observar tres grandes grupos que están representadas en las siete piezas del teatro $n \bar{o} .^{14}$

La primera categoría es la de una poeta representativa de Heian, como se refleja en Sōshiarai Komachi (草子洗い小町). ${ }^{15}$ En esta obra la imagen de Komachi es la de la poetisa triunfante superior al rival al quien se enfrenta en la competición de waka en la corte. La historia cuenta que Ōtomo no Kuronushi, uno de los Rokkasen (六歌仙, Seis santos de la poesía) elegidos por el compilador de Kokinshū, Ki no Tsuyayuki, intenta burlarse de Komachi y humillarla haciendo trampa. Su estrategia consiste en espiar a Komachi mientras ésta compone su poema. Lo escribirá en un libro de Man'yōshū como si de antiguo poema se tratase. El día de la lectura, Kuronushi la denuncia como plagiadora delante de todos los cortesanos. Sin embargo, Komachi responde a esta maldad pidiendo que lavase la página con el agua, lo que causa el corrimiento de la tinta y el poema desaparece. Así se demuestra que se trata de una acusación falsa y Komachi es alabada y reconocida como gran poetisa.

A esta categoría pertenece otra pieza en la que se inspiraron múltiples obras del género de literatura oral como nagauta, kiyomoto, ${ }^{16}$ jōruri ${ }^{17}$ y kabuki en la etapa de Edo. Se titula Amagoi Komachi (雨乞W小町, Komachi, imploradora de lluvia). ${ }^{18}$ Aunque, como sí ocurría en el caso de la pieza de teatro $n \bar{o}$, ya no existe el guion escrito, tenemos suficiente referencia histórica de la obra. El descendiente de Motoori Norinaga, ${ }^{19}$ Motoori Uchitō ${ }^{20}$ señala un poema de la colección Komachish $\bar{u}^{21}$ como la composición que se puede atribuir a la ocasión. En esta historia Komachi se encuentra fuera de la corte y lleva una vida triste y solitaria. Sin embargo, la fama de excelencia de la poetisa sigue viva en la mente del emperador y, al no encontrar remedio mejor para subsanar la sequía prolongada que sufre el país, envía un emisario a Komachi para que haga el uso de su poder milagroso con su poema. La poetisa accede a la petición del soberano y declama el siguiente poema:

\section{千早振る神も見まさば立ち騒ぎ天の戸川の樋口あけ給へ}

Chihayaburu kami mo mimasaba tachisawagi ama no togawa no higuchi aketamae ¡Oh, grandiosos dioses del cielo!

13 Terasaki, Etsuko (1984): "Images and Symbols in Sotoba Komachi: A Critical Analysis of a Nō Play". En Harvard Journal of Asiatic Studies, vol. 44, nº. 1, June, pp. 155-184. Disponible en: http://www.jstor.org/stable/2719097 [Consulta: 09/10/2014].

14 Las siete piezas son Kayoi Komachi, Sotoba Komachi, Oumu Komachi, Sekidera Komachi, Amagoi Komachi, Soushiarai Komachi y Kiyomizu Komachi.

15 Su autor es Zeami o en algunas versiones, Kan'ami. Sōshiarai Komachi (Komachi que lava el libro). Kanzeryū keikoyō uaibon, 3 de 6 (edición para las prácticas de la escuela Kanze). Tokio: Keyaki shoten, 1955.

16 https://ci.nii.ac.jp/books/search?advanced=false\&l=ja\&q=雨乞い小町 [Consulta: 02/02/2018].

17 Torii, Fumiko (1987): “土佐浄瑠璃の脚色法 一小町もの「吾妻業平色小町」「桜小町」-Adaptations of Tosa Joruri for the Stage (IX)-: Two Komachi-mono: ‘Azuma Narihira Iro Komachi' and 'Sakura Komachi'”. En: Essays and Studies, 38 (1), Tokio, pp. 109-140. Disponible en http://ci.nii.ac.jp/naid/110006000446 [Consulta: 18/02/2015].

18 También es conocida como Takayasu Komachi (高安小町). Hangyo’s Archive, Kanazawa, Disponible en http:// hangyo.sakura.ne.jp/utahi/text/yob21.txt [Consulta: 02/02/2018].

19 Motoori Norinaga (本居宣長) (1730-1801)

20 Motoori Uchitō (本居内遠) (1792-1885) Segunda cabeza de familia Motoori. Fue nieto adoptivo de Motoori.

21 Komachishū (小町集) Seguramente se compiló hacia finales del siglo IX después de la muerte de Komachi y recoge más de 100 poemas incluyendo los seleccionados en Kokinwakashū. 


\begin{abstract}
Si contempláis esta tierra seca, ¡que os levantéis a recorrerla sobre las nubes para abrir la llave del río del cielo!
\end{abstract}

La rogativa hace efecto y se produce un diluvio sobre la tierra. En esta leyenda se revela la atribución del poder mágico de las palabras conocido como kotodama, ${ }^{22}$ así como la capacidad de la poetisa para obrar milagros. Se detecta en este sentido la creencia ancestral del poder de las miko, sacerdotisas sintoístas (incluyendo a Komachi entre ellas), que han dejado diferentes testimonios a lo largo de la historia japonesa.

La segunda categoría es la de una mujer fría e insidiosa reflejada en Kayoi Komachi (通い小町). Esta obra de teatro nō recoge la relación turbulenta entre su pretendiente, el capitán Fukausa, y Komachi. Ante la petición de mano del capitán, Komachi reacciona con frialdad. Le pide visitarla todas las noches hasta cien para acceder a su deseo, pero el último día para cumplir con la promesa, el capitán cae enfermo $^{23}$ y no puede acudir al palacio de Komachi. La obra de teatro $n \bar{o}$ presenta al fantasma del capitán que expresa su rencor hacia Komachi e intenta vengarse de ella en la escena, pero gracias a la ayuda de las plegarias del bonzo que visita el lugar, los dos consiguen alcanzar la paz espiritual.

Esta historia tiene una estrecha relación con la imagen de femme fatale que se propagó a través de otras obras posteriores. ${ }^{24}$ Sin embargo, en realidad es una historia que aparece como comentario explicativo de un poema en Kokinwakash $\bar{u}^{25}$ y, originalmente, no tenía relación alguna con Komachi. Se entiende que la tendencia de usar la legendaria imagen de Komachi, que rechaza a sus pretendientes habría empujado a narrar el relato como si fuera un hecho que ocurrió en su vida.

En relación con este grupo de obras, además de esta consideración, sería relevante tener en cuenta la anteriormente mencionada Tamatsukuri Komachishi Sōsuisho del siglo X. ya que se puede considerar un antecedente para la creación de tal leyenda.

La tercera categoría sería la que la representa con la figura de una vieja pordiosera, como en Sotoba Komachi (卒塔婆小町) ${ }^{26}$ y en Sekidera Komachi (関寺小町) ${ }^{27}$ En ambas obras Komachi se presenta como una mujer mayor y fea, totalmente des-

22 Takagi, Kayoko (2004): El cuento del cortador de bambú, Letras Universales 370. Madrid: Cátedra, pp. 2851. Takagi, Kayoko (2010): ““El concepto del ánima de las palabras, kotodama en la literatura japonesa”. En: LYNX, A monographic Series in Linguistics and World Perception, Lenguas de Asia Oriental: Estudios Lingüisticos y Discursivos, Annexa 18, Valencia, Universidad de Valencia, pp. 263-278.

23 En la versión de Kokinwakashū se muere el padre del capitán y no puede acudir a visitar a Komachi.

24 Strong, Sarah, M. (1994): "The Making of a Femme Fatale. Ono no Komachi in the Early Medieval Commentaries". En Monumenta Nipponica, vol. 49, no. 4, Winter, pp. 391-412. Disponible en: http://www.jstor.org/ stable/2385255 [Consulta: 17/02/2014].

25 Takagi, Kayoko. y Janés, Clara (2008): 9 piezas..., op. cit, p. 185. Además, encontramos un poema que podría bien poseer una estrecha relación con esta historia en Kokinwakashū (Poema $\mathrm{n}^{\circ}$ 522). En este caso la prueba consiste en visitarla cien noches seguidas y escribir el número en la superficie del agua del río. Strong, Sarah. M. (1994): "The Making of..., op. cit., pp.407- 408.

26 Koyama, Hiroshi y Sato, Kenichiro (eds.) (2003): Nihon bungaku koten zenshū (日本古典文学全集59, Yōkyokushū, 謡曲集2). Tokio: Shogakukan, pp. 116-127.

27 http://base1.nijl.ac.jp/iview/Frame.jsp?DB_ID=G0003917KTM\&C_CODE=0098-001701-212http://base1. nijl.ac.jp/iview/Frame.jsp?DB_ID=G0003917KTM\&C_CODE=0098-001701-212 [Consulta: 17/02/2014] La época es la de la fiesta de las estrellas Tanabata (七鸟) y, a petición del prior del bonzo, Komachi se presta a hablar de su pasado brillante y sobre las esencias del poema japonés. La obra termina con un dificultoso baile de la anciana que intenta recuperar la memoria de la gloria y del placer del pasado. 
provista del lujo de antaño. Aunque la belleza y el garbo habían desaparecido de su rostro, su elegancia innata y su sabiduría poética siguen vivas y es capaz de orientar a la gente con sus palabras.

Sin que se haya podido probar con datos históricos, se ha extendido esta fama de la precaria vida de Komachi que en su vejez deambulaba por las calles de Kioto y por los caminos de la región del Norte. Esta leyenda es la que ha inspirado a diferentes narraciones llamadas Komachi zŌshi (小町草子) ${ }^{28}$ producidas en el período Muromachi, en las que la calavera de Komachi llama al poeta Ariwara no Narihira. El gusto popular creó esta relación amorosa entre dos de los personajes más destacados de la época de Heian y la identificación de la calavera con la muerte de Komachi en absoluto abandono se repitió en muchas obras posteriores. ${ }^{29}$

Tras el recorrido por las obras relacionadas con Komachi hasta el período Edo, parecía que los temas sobre la figura estaban prácticamente agotados. Sin embargo, en la era de Meiji se publicó un libro titulado Ono no Komachi-ron, escrito por uno de los líderes de opinión de la época, Kuroiwa Ruikō (1862-1920) y reeditado en $1994 .^{30}$ Según el argumento de Kuroiwa, para el movimiento feminista de la época que deseaba impulsar, la figura de Komachi era modélica, pues consiguió mantenerse soltera por sus principios individualistas.

Si el progreso de la sociedad durante las eras Meiji y Taishō ensalzó a Komachi en un modo novedoso, en la etapa más moderna también ha habudi escritores que se han inspirado en ella. Mishima Yukio (1925-1970) escribió una pieza del $n \bar{o}$ moderno con el mismo nombre que la obra de Zeami, Sotoba Komachi ${ }^{31}$ Mishima traza la historia entre una vieja que recoge colillas de tabaco en un parque y un poeta joven que habla con ella. El contraste de vitalidad y vejez, destino y tragedia están magistralmente entretejidos en esta obra. Otra novela inspirada en Komachi de nuestros tiempos es Komachi Hensō, de Enchi Fumiko. ${ }^{32}$ La autora desarrolla una historia sobre la problemática universal de la mujer como es la infertilidad y la vejez, o la pérdida de la lozanía juvenil. En nuestros tiempos la producción artística inspirada en Komachi tampoco cesa de aparecer en obras de variado género como en el teatro, la música o en el manga y anime.

\section{Dispersión geográfica del nombre del clan Ono y de Komachi}

Como hemos observado hasta aquí, dada la importancia de la tradición literaria que atañe a la poetisa, no es de extrañar que los lugares geográficos que ostentan nom-

28 Existen varias versiones con el mismo nombre. Una de ellas está recogida en Ichiko, Teiji (ed.) (1994): Otogi$z \bar{o}$ shi (御伽草紙) 上 (T. I). Tokio: Iwanami shoten, pp.87-105.

A partir del período Kamakura (s. XIII-XIV) empezaron a aparecer colecciones de cuentos y relatos populares en formato corto y normalmente con dibujos. Se les llama Otogizōshi. Este género evolucionaría hasta la etapa premoderna y sirvió en gran medida para el conocimiento de leyendas y relatos históricos de la gente de a pie.

29 Torii, F. (1987): “土佐浄瑠璃の脚色法 一小町もの「吾妻業平色小町」「桜小町」-Adaptations of Tosa Joruri for the Stage (IX)-: Two Komachi-mono: 'Azuma Narihira Iro Komachi' and 'Sakura Komachi'”, op. cit.

30 Kuroiwa, Ruiko (1994): Ono no Komachi ron (小野小町論). Tokio: Shakai shisōsha.

31 Mishima, Yukio (2014): “Sotoba Komachi”. En Kindai Nôgaku-shû (近代能楽集). Tokio: Shinchō bunko, (53ª ed.), pp. 94-118. Existe la traducción al español de Kazuya Sakai (traductor) (1959): La mujer del abanico. Seis piezas del teatro moderno. Buenos Aires: Mandrágora.

32 Enchi, Fumiko (1982): Komachi hensou (小町変相) (El disfraz de Komachi). Tokio: Shueisha bunko ( $8^{\mathrm{a}}$ ed.). 
bre de Ono reclamen hoy su relación vital con la literata. Solamente rastreando el mapa de Japón con el propósito de buscar la tumba de Komachi, encontramos una dispersión amplia desde el norte hasta el sur. Se trata de las prefecturas de Akita, Miyagi, Fukushima, Tochigi, Ibaraki, Kioto, Shiga, Tottori, Okayama, Yamaguchi y Kumamoto. ${ }^{33}$ Sin embargo, por razón del linaje a partir del famoso político Ono no Imoko, como personaje más conocido de la historia, hasta Ono no Takamura, ${ }^{34}$ como familiar más cercano a la figura de Komachi (que se debate si es el padre o el abuelo de ella), la prefectura de Shiga parece destacar con más relevancia al hablar del clan $\mathrm{Ono}^{35}$. A pesar de este hecho, desde el punto de vista del nacimiento de Komachi, no se puede descartar la relación que tuvo Takamura con la región de Mutsu, ${ }^{36}$ que comprendía una parte de la actual prefectura de Akita. El padre de Takamura fue gobernador de Mutsu y Takamura mismo ocupó el cargo desde el 842. Por su parte, en la villa Ono de la prefectura de Fukushima se conservan las ruinas del palacio de Takamura y en la placa oficial de la villa se lee que Ono no Minemura junto con su hijo Takamura contribuyeron a la prosperidad del lugar a través de transmisión de conocimientos de la cultura Heian desde la capital Kioto hasta allí. ${ }^{37}$

Esta transmisión cultural no solo ocurrió en etapa tan remota como ésta, sino también en una era muy posterior como la de las guerras civiles, conocida como época de Sengoku del siglo XVI. A fin de corroborar esta afirmación, me parece relevante observar la función que cumplía la figura de Ono O-tsū bastantes siglos más tarde desde esta época hasta el inicio del bakufu de Tokugawa (1568-1672) y dio motivos para más investigaciones históricas..$^{38}$ Ono O-tsū aparece en varios documentos como mujer elegante, artista y poetisa de Kioto, que enseñó el protocolo de Heian a las esposas e hijas de Nobunaga, Hideyoshi, Ieyasu, Iemitsu hasta Tokugawa Hidetada. ${ }^{39}$ Por la extensión en el tiempo que abarcan estos nombres y que dura más de 100 años, se considera que eran dos o más mujeres con el mismo nombre, seguramente, madre e hija o hijas ${ }^{40}$ Los documentos recientemente revelados de la casa del clan Sanada ${ }^{41}$, especialmente las cartas intercambiadas entre Sanada Nobuyuki ${ }^{42}$

33 Prefectura de Akita, AKITA WEB tourist information, http://www.akitabi.com/, Disponible en http://komatide. web.fc2.com/mappu.html. [Consulta: 17/08/2016]

34 Ono no Takamura (小野筌) (802-852) famoso político, poeta, erudito de principios de la era Heian.

35 Vv.Aa. (1990): Nihon rekishi jinmei jiten (歴史人名辞典). Tokio: Kodansha gakujutsu bunko, pp.38 y 183. Existe la comarca Ono donde se hallan los santuarios con los nombres de Ono y Takamura. https://ja.wikipedia. org/wiki/小野村_(秋田県) [Consulta: 17/08/2016]

36 Comprende un vasto territorio que incluía las actuales prefecturas de Fukushima, Miyagi, Iwate, Aomori y parte de Akita. El padre de Takamura, Minemori (岑守, 778-830) fue gobernador de esta región, desde 815 hasta 822 , y Takamura vivió también allí en su juventud.

37 http://komatide.web.fc2.com/gazo/fukuono/yakata.html [Consulta: 17/08/2016].

38 Kasahara Hisako (2001): Onoke no on'natachi, op. cit. pp. 145-147.

39 Ibid., pp.144-145.

40 Popularmente, se le atribuye también la creación de jōruri, cosa que está descartada por los historiadores, pero el nombre de Ono Otsu quedó en la historia como la mujer que recitó a Nobunaga la historia de la princesa Jōruri, la primera obra del género jōruri.

${ }^{41}$ Kasahara Hisako (2001): Onoke no on'natachi, op. cit., p. 151. Las cartas intercambiadas entre Ono Otsu y la casa Sanada están recogidas en Sanada Toshiko (1990): Ono Otsu: Sanada Kageyuke monjokara (小野捄通: 真田勘解由家文書から). Tokio: Editorial Fukeisha, 1990. Con ellas se han comprobado la existencia de la poeta perteneciente al clan Ono a la vez que su estrecha relación con el clan Sanada.

42 Sanada Nobuyuki 真田信之 (1566-1658). Luchó por el bando de Tokugawa en la batalla de Sekigahara contra la banda de Ishida Mitsunari (tras la muerte de Hideyoshi) a la que se había unido su padre Masayuki y su hermano pequeño Nobushige (popularmente conocido como Yukimura). 
y su hijo Nobumasa y O-tsū, han confirmado que su contribución como enlace entre la clase noble de Kioto y la clase guerrera bushi fue decisiva en diferentes ocasiones históricas. ${ }^{43}$

Como Kasahara concluye afirmativamente, O-tsū puede considerarse descendiente del linaje femenino de la familia Ono que se caracteriza por el afamado talento artístico de Komachi desde el período Heian. A propósito, Yanagita Kunio había escrito un artículo en 1939 sobre esta figura y la calificaba de mujer dentro de la tradición de las kataribes andantes que viajaban por diferentes regiones contando las memorias de su familia y sugería la línea genealógica del linaje Ono desde Komachi hasta O-tsū. ${ }^{44}$

\section{Ogachi hoy}

Uno de los lugares que hace alarde de ser el sitio de nacimiento de Komachi es la villa de Ogachi en Akita, donde podemos encontrar varios monumentos y ruinas que guardan la memoria de la vida y las leyendas sobre Komachi. A fin de conocer y examinar directamente dicha memoria histórica, tomé el nuevo shinkansen [fig. 2], a Akita, diseñado en color rojo con el nombre de Komachi, hasta Ōmagari y luego seguí en la línea de Ōu hasta la estación de Yokobori, pasando por Yuzawa. El pueblo donde custodian dichas memorias ostenta el nombre de Ono-mura (Poblado de Ono) que se fusionó en 1955 con el área actual de Ogachi.

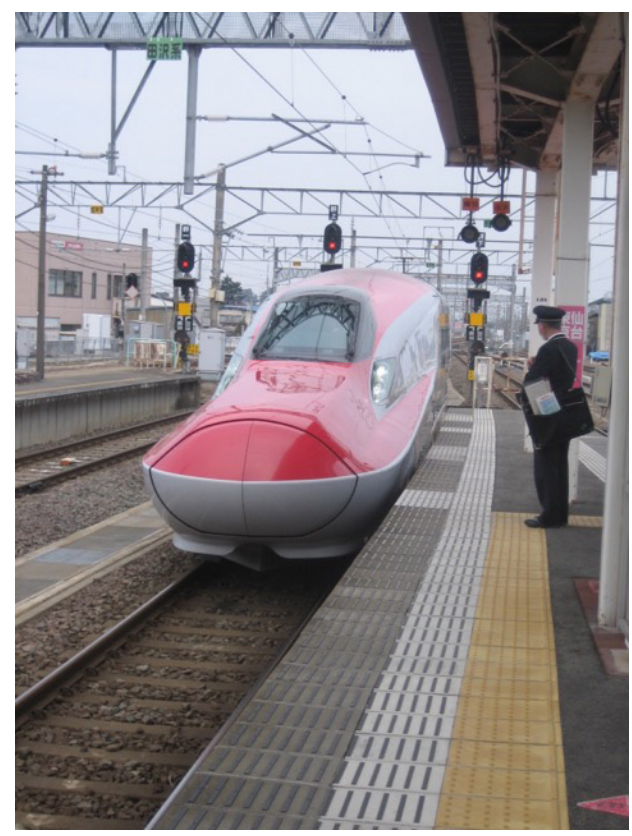

Fig. 2: Nuevo modelo de shinkansen denominado Komachi, JR Higashinihon. (C) 2016, Kayoko Takagi.

43 Kasahara Hisako (2001): Onoke no on 'natachi, op. cit., pp. 144-220.

44 Yanagita, Kunio (1942): “Ono O-tsū”. En: Imo no chikara (妹の力). Tokio: Kadokawa, pp.260-285. 
La mención más antigua que se conoce a tal atribución aparece en el siglo XVII en el antes citado género, gunki monogatari (軍記物語, crónicas de guerra), en el que se describen las batallas y guerras habidas en la zona septentrional de Honshu desde la etapa de guerras civiles hasta la constitución del bakufu en Edo. ${ }^{45} \mathrm{El}$ autor Tobe Ikkansai (1645-1708) era de Ogachi por lo que Nishiki determina que, al menos, en su época desde finales del siglo XVI a principios del XVII, era compartido entre los habitantes el conocimiento sobre las ruinas del palacio del supuesto padre de Komachi, Yoshizane. ${ }^{46}$ Aunque en la genealogía del clan Ono aparece su nombre y varios carteles turísticos organizados oficialmente por el ayuntamiento de Ogachi la mencionan, su verdadera existencia es incierta por carecer completamente de referencia en los documentos históricos. Por otra parte, entre otros escritos que recogen dichas memorias a partir de esta época, hemos mencionado los de Sugae Masumi (1754-1829), un cronista de viajes que escribió sobre sus visitas a la zona entre 1785 y 1822. El propósito del viaje de Sugae fue claro: seguir y comprobar los antiguos pasos de Ono no Komachi en Ogachi. Como fruto de estas visitas al lugar llegó a escribir relatos sobre la vida de la poetisa que le habían contado los habitantes del lugar. ${ }^{47}$

\section{El desarrollo de leyendas y sus memorias}

Nishiki traza los pasos de Sugae e investiga los templos relacionados con la vida de Komachi en Ogachi. Su investigación llega a determinar el foco de propagación de las leyendas sobre Komachi en un templo de shugendō, ${ }^{48}$ llamado Kakugen'in, actual santuario de Kumano. En la placa turística delante del templo podemos leer una explicación somera del monumento, indicando que fue construido por el padre de Komachi Yoshizane en 802, y en uno de los pabellones custodiaba una poesía compuesta por Komachi. Tras sufrir incendio total por las batallas en la zona, fue reconstruido en 1672 por Kakugen'in. Mención a varias poesías waka atribuidas a la poeta no faltan. Cerca de los templos se divisan dos montículos que se llaman Futatsumori (Dos bosques), que se supone contienen las tumbas de Komachi y su pretendiente el capitán Fukakusa. Como leemos en la pieza de Kayoi Komachi, el capitán visitó a Komachi durante noventa y nueve noches, pero en la última noche falló y murió sin conseguir su propósito.

Tomando como centro el palacio Ono, en un radio de 2 kilómetros se encuentran prácticamente todos los monumentos y ruinas en relación a Komachi. Pude comprobar que los monumentos estaban todos referidos en las diferentes obras literarias arriba mencionadas:

1. Dos pequeñas colinas que se les llaman Futatsumori.

2. El pozo hexagonal al estilo Heian. Se dice que se utilizó su agua para bañar a Komachi recién nacida.

\footnotetext{
Nishiki, Hiroshi (2004): “Ōu eikei gunki” (奥羽永慶軍記). En Ono no Komachi..., op. cit., p.16.

小野良実. Vv.Aa. (1990): Nihon rekishi..., op. cit., pp. 38 y 183.

Ono no Komachi..., op. cit., pp. 38-44.

48 Shugendō (修験道). Es una especie de ascetismo de montaña japonés que sincretiza la enseñanza budista con la sintoísta.
} 
3. La tumba de la madre de Komachi dentro del supuesto recinto del palacio. En varias piedras se leen algunos textos de Sutras.

4. El santuario de Kumano, que se llamaba Kakugen'in.

5. El templo Ono que hoy se llama Kōyaji, donde custodian una escultura de madera cuya autoría se le atribuye a Komachi antes de su muerte. ${ }^{49}[\text { fig. } 3]^{50}$

6. El templo Chōsen-ji en el que se hospedó el capitán Fukakusa durante 99 noches para cortejar a Komachi.

7. El puente de donde supuestamente cayó al río el capitán y murió trágicamente.

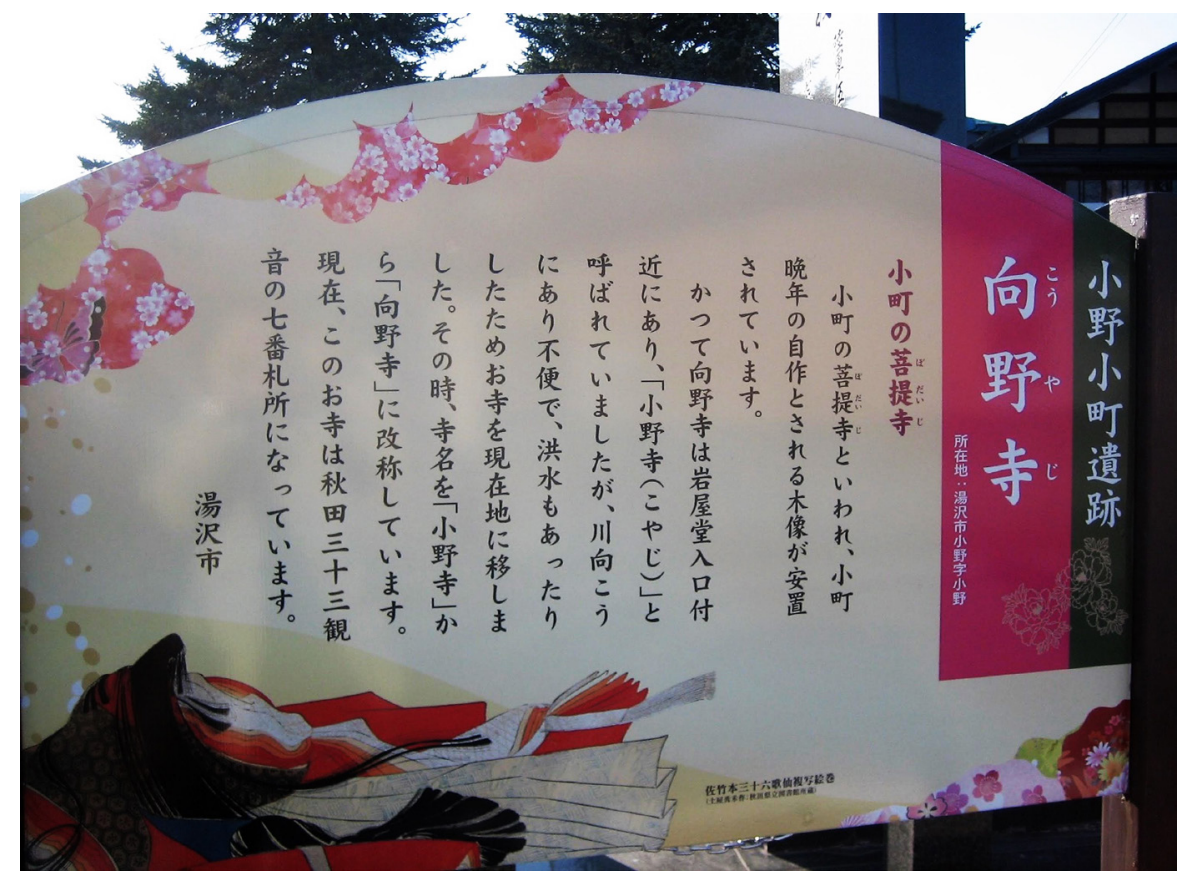

Fig. 3: Cartel turístico, Villa de Ogachi, Ayuntamiento de la ciudad de Yuzawa. En el cartel, aparece una reproducción de la figura de Komachi sacada de Satake-bon Sanjürokkasen fukusha emaki (佐竹本三十六歌仙複写絵巻, Copia de 36 poetas selectos de la compilación Satake. Copista: Tsuchiya S., 1901, Biblioteca Prefectural de Akita. (C) 2016, Kayoko Takagi.

49 La imagen de Komachi envejecida se puede ver también en el templo Fudaraku-ji de Kioto, pero este tipo de obra relacionada con Komachi se encuentra en diferentes lugares del territorio japonés.

Centro para Convenciones y Visitantes Turísticos de Kioto, Kyoto Kanko Navi Oficial site, http://kanko. city.kyoto.lg.jp, 2007, disponible en: http://kanko.city.kyoto.lg.jp/detail.php?InforKindCode=4\&ManageCo$\mathrm{de}=1000200$. [Consulta: 30/03/2016]

50 La información escrita en el cartel de la fotografía es la siguiente:

"Kōyaji ubicación: Comarca de Ono de la Ciudad de Yuzawa.

Templo de Komachi: Este templo se considera el templo donde se enterró el cuerpo de Komachi y dentro se custodia una talla de madera cuya autoría es atribuida a las manos de Komachi en la etapa final de su vida.

Hace tiempo el templo de Kōyaji se encontraba cerca de la entrada a una cueva y se llamaba Kōyaji. Sin embargo, como estaba más allá del río, el acceso era difícil. Además, sufrió alguna inundación en la época por lo que lo trasladaron a este lugar. En ese momento, el nombre se cambió por Kōyaji (高野寺) en lugar de Kōyaji (小 野寺). Actualmente, este templo es uno de los treinta y tres sitios de peregrinación de Kannon de la región de Akita. Ciudad de Yuzawa." 
Los carteles en cada monumento destacan su relación histórica con la poetisa sin revelar realmente cuál es la credibilidad de los hechos. Existe un pabellón en honor a Komachi donde se celebra anualmente un concurso de waka. Aunque no es un monumento histórico, los turistas de hoy lo visitan para rendir homenaje a la figura de Komachi. Además, en la misma zona el ayuntamiento está construyendo un parque temático, sin terminar todavía en 2017, teniendo como eje central la figura de Komachi.

Lo que no pude comprobar con mis propios ojos en esta visita al lugar fue el jardín de peonías que se relaciona con el poder mágico de la poetisa, cuando fue capaz de provocar lluvia con su poema. La razón fue que había un plan de incorporar ese jardín dentro del parque temático y aún no habían terminado de trasladar los bulbos de peonías desde el anterior jardín. Esta leyenda, como mencionamos anteriormente, está recogida en la pieza del teatro nō Amagoi Komachi (雨乞い小町) creada por Zeami. Sugae a finales del siglo XVIII lo visitó y describió como un jardín especial, que se cuidaba con la firme creencia en el milagro obrado en su día. Para comenzar la plantación del arroz se tomaba como referencia temporal la floración de las peonías y se organizaba una rogativa para la lluvia y una romería colocando una imagen de la poetisa con varios tallos de peonía doblados, signo que se creía que provocaba mayor cantidad de lluvia. ${ }^{51}$

\section{Conclusión}

Como señalaba Yanagita Kunio, las leyendas son los relatos que quieren ser parte de la historia. ${ }^{52}$ Las tres caras principales de la figura de Komachi están representadas como memoria colectiva de Ogachi y nos invitan a soñar con un pasado incierto. Hoy la administración de Yuzawa y la de la prefectura de Akita desarrollan una campaña turística con el uso de dichas evocaciones remotas. ${ }^{53}$ Como se desprende naturalmente de la memoria de la poetisa de Heian, su herencia literaria se palpa en diferentes rincones de la zona. Anualmente, las obras de poesía waka que reciben mención en ese concurso en homenaje a Komachi pueden exponerse en el pabellón de Komachi durante un año. Para añadir otra razón más sobre esta transmisión cultural desde el centro a la periferia, no debo omitir los recuerdos del viaje de Bashō por el norte de Japón que dejó una importante influencia en los caminos de Oku. En este sentido, para un viajero que llega desde Tokio, sorprende la custodia y el desarrollo de la literatura clásica en esta región boreal del país.

Por otra parte, encontramos, sin buscar demasiado, gran frecuencia y diversidad de objetos y fenómenos que llevan el nombre de Komachi. Entre ellos destacan marcas comerciales, siendo la más popular, la denominación del nuevo shinkansen a Akita y las de productos típicos de esta prefectura: Akitakomachi y Awayukikoma-

Nishiki, Hitoshi (2004): Ono no Komachi, op. cit., pp. 59-63.

52 Yanagita, Kunio (1990): Densetsu (伝説) (leyendas). En: Yanagida Kuniko Zenshu (柳田國男全集 ) (Colección Yanagita Kunio), vol. 7, Tokio: Chikuma bunko, p. 36.

53 Página oficial de la ciudad de Yuzawa, Disponible en http://www.city-yuzawa.jp/midokoro04/1133. html [Consulta: 26/01/2018] 
chi para el arroz ${ }^{54}$ o Fukukomachi para el sake ${ }^{55}$. De este modo, se comprueba con claridad que el aprovechamiento de la tradición cultural de la zona está estrechamente relacionado tanto con las campañas comerciales como con la imagen romántica del pasado de esta área.

La prefectura de Akita hace uso de sus monumentos y ruinas para potenciar la riqueza folklórica de la región, a la vez que sus posibilidades comerciales y turísticas. La fotografía publicitaria de una bella joven con el sombrero de paja que se cuelga en las estaciones de shinkansen ${ }^{56}$ [fig. 4], recuerda a la perfección la figura legendaria de Komachi habiéndose convertido en la imagen identitaria del lugar. El tren "bala" Komachi que se inauguró en 1997 lleva cada día a miles de turistas hacia esa región para recordar la memoria de su patrimonio cultural, que permite soñar con el pasado elegante de la poeta de Heian.

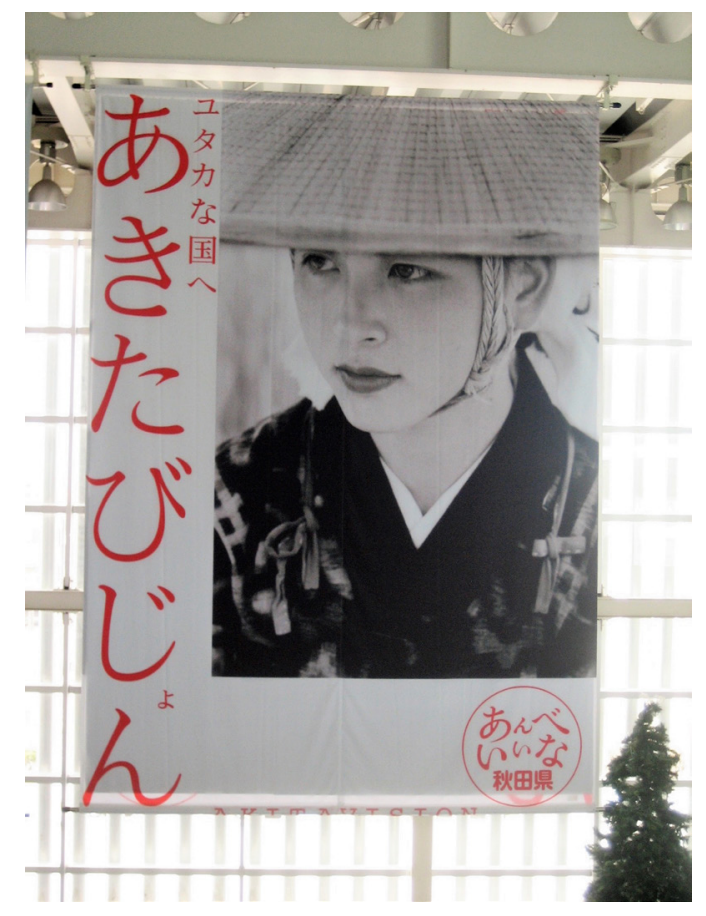

Fig. 4: Akita bijon, foto publicitaria (2015). Estación de Oomagari, Sección de estrategia turística, Departamento de turismo, cultura y deporte de la Prefectura de Akita. (C) 2016, Kayoko Takagi.

54 Zen'nō Akita, Sede en Akita de la Asociación cooperativa de agricultura nacional. Disponible en: http://www. ak.zennoh.or.jp/rice/lineup.html [Consulta: 26/01/2018]

55 Kimura shuzō, 2017, Fukukomachi, [http://www.fukukomachi.com]. Disponible en: http://www.fukukomachi. com/item/index.html [Consulta: 26/01/2018]

56 Iphone Smaho kyōshitsu/Sapporo, Mina, 2017. Disponible en:https://halmina.jp/kimuraihei-akita/ [Consulta: 30/03/2016], Es obra de Kimura Ihei (木村伊兵衛, 1901-1974), uno de los fotógrafos más valorados de nuestro tiempo. Realizó fotos sobre la región de Akita y esta obra Akita obako (Belleza de Akita producida en 1953) forma parte de esta colección. Actualmente, la obra está siendo utilizada como imagen publicitaria de Akita con el título Akita bijon (秋田びじょん) que hace juego de palabras de bijin (美人) con bijon (visión o imagen). 